\title{
Ramp Forecasting Performance from Improved Short- Term Wind Power Forecasting Over Multiple Spatial and Temporal Scales
}

\author{
Jie Zhang ${ }^{\mathrm{a}, 1}$, Mingjian Cui ${ }^{\mathrm{a}}$, Bri-Mathias Hodge ${ }^{\mathrm{b}}$, Anthony Florita ${ }^{\mathrm{b}}$, Jeffrey \\ Freedman ${ }^{\mathrm{c}}$ \\ ${ }^{a}$ University of Texas at Dallas, Richardson, TX, USA \\ ${ }^{b}$ National Renewable Energy Laboratory, Golden, CO, USA \\ ${ }^{c}$ State University of New York at Albany, Albany, NY, USA
}

\begin{abstract}
The large variability and uncertainty in wind power generation present a concern to power system operators, especially given the increasing amounts of wind power being integrated into the electric power system. Large ramps, one of the biggest concerns, can significantly influence system economics and reliability. The Wind Forecast Improvement Project (WFIP) was to improve the accuracy of forecasts and to evaluate the economic benefits of these improvements to grid operators. This paper evaluates the ramp forecasting accuracy gained by improving the performance of short-term wind power forecasting. This study focuses on the WFIP southern study region, which encompasses most of the Electric Reliability Council of Texas (ERCOT) territory, to compare the experimental WFIP forecasts to the existing short-term wind power forecasts (used at ERCOT) at multiple spatial and temporal scales. The study employs four significant wind power ramps definitions according to the power change magnitude, direction, and duration. The optimized swinging door algorithm is adopted to extract ramp events from actual and forecasted wind power time series. The results show that the experimental WFIP forecasts improve the accuracy of the wind power ramp forecasting. This improvement can result in substantial costs savings and power system reliability enhancements.
\end{abstract}

Keywords: Wind forecasting; grid integration; ramp forecasting; ERCOT; optimized swinging door algorithm

\section{Introduction}

As an important renewable energy resource, wind power has been increasing dramatically in the electric power system. Currently, wind power meets approximately $4 \%$ of U.S. electricity demand [1]. However, some systems have noticeably higher amounts of wind installed; for example, the Electric Reliability Council of Texas (ERCOT) has experienced instantaneous wind power penetrations up to 29\% [2]. Xcel Energy's Colorado system reached $60.5 \%$ hourly penetration [3]. The characteristics of upper availability limit, large variability, and uncertainty in wind power present a primary concern to system operators. Especially given the increasing amounts of wind power being integrated into the electric power system, accurate wind power forecasts are critically important for reliable and economic power system operations [4-6]. Although the current power grid is capable of handling small amounts of uncertainty and variability, ramp (or extreme) events - such as sudden and large changes in wind power-are a critical concern for the system with high wind penetration. Improving the accuracy of wind power forecasting is expected to reduce the discrepancy between the forecast and actual wind power output, and thereby enhance the performance of wind power ramp forecasting and reduce wind integration costs.

\subsection{Wind Power Ramp Forecasting}

${ }^{1}$ Corresponding author. Tel.: +1-303-384-6981

E-mail address: jiezhang@utdallas.edu (Jie Zhang) 
Large fluctuation incidents with large magnitudes and short durations, so-called ramping events, are a significant concern of power system operators. One of the biggest concerns associated with integrating a large amount of wind power into the grid is the ability to forecast and handle large ramps in wind power output. ERCOT has been experiencing a rapid growth of installed wind generation capacity and this tendency is expected to continue growing into 2017 [7]. A large down-ramp event occurred in the ERCOT system on February 26, 2008, that caused a system emergency [8]. Different time and geographic scales influence wind ramps, and both up and down ramps can have varying levels of severity. A similar challenge is also encountered in other power systems experiencing the rapid growth of renewable resources, e.g., California (Duck Curve [9]) and Hawaii. As the recent study conducted by the National Renewable Energy Laboratory (NREL) explored the implications and challenges of very high renewable electricity generation levels - from $30 \%$ up to $90 \%$, focusing on $80 \%$, of all U.S. electricity generation - in 2050 , one great obstacle needed to solve for such a high penetration of renewables is to predict the ramping events accurately and understand their implications on the power system operation [10].

Different time and geographic scales influence wind ramps. Up and down ramps can have varying levels of severity, and generally, a down ramp could be more risky than an up ramp because of the availability of reserves. There are generally two main ways in which an inaccurate forecasting of ramp events can lead to large errors: ramp magnitude and timing errors. A magnitude error is defined as an event that is forecasted to occur at an expected time but with significantly different magnitude. In a ramp timing error, the actual ramp in power significantly leads/lags the forecasted ramp time. A correct forecast of large wind and solar ramps, especially about when they will happen, is imperative to reduce the wind integration costs and to ensure the reliability and security at independent system operators (ISOs). For example, an operator could decide to commit fewer MWs of fast-start units due to confidence on the near-term wind ramping capability forecasts, ultimately resulting in reduced system operation costs and increased system reliability.

Ramp forecasting is a relatively recent topic motivated by the need of improving the management of large and fast wind and solar power output variations, especially in a context of power systems with high renewable penetration. Thus, what constitutes the optimal output of a ramp-oriented forecasting tool is still not well defined. Generally, ramping events are parameterized by the following properties: ramping start/end, ramping duration, ramping rate, and ramping magnitude. Ramp event alerts, probabilistic ramp event occurrence, and ramp rate forecast represent some examples of the different ramp forecasting standpoints [11]. Ferreria et al. [12] provided an overview of different ramp definitions and approaches to ramp event forecasting. Greaves et al. [13] defined a ramp as a change in wind power output that is at least $50 \%$ of the installed wind capacity and occurs within a time span of 4 hours or less. Zheng and Kusiak [14] employed the rate of change of wind power output during a 10-minute interval to define a ramp. Potter et al. [15] defined a ramp event as the change in power between two consecutive hours that is greater than or equal to $10 \%$ of the installed wind capacity. In a report by AWS Truewind [16], the up and down ramps were differently defined considering the different levels of risk: (i) a down ramp occurs if the power changes at least $15 \%$ of total capacity within one hour; and (ii) an up ramp occurs if the power changes at least $20 \%$ of total capacity within 1 hour. AWS Truewind also analyzed the ERCOT system, which defines a ramp event as a change of $20 \%$ or more of the rated capacity in any 30 -minute period [17]. It is important to note that a variety of ramp definitions in terms of capacity and temporal differences are commonly used in power systems.

Ramp forecasting can be characterized into two categories: indirect and direct forecasts. For indirect ramp forecasting, wind power forecasts can be produced by multiple forecasting methods such as numerical weather prediction (NWP), statistical, and machine learning methods. Ramp detection methods could be adopted in a postprocessing method to detect ramping events in the forecasted wind and solar power. Both deterministic and probabilistic ramp forecasts can be produced in this way. For direct ramp forecasting, ramp extraction methods can directly be applied to historical measured and forecasted wind power to extract all historical ramping events. Statistical and machine learning methods can be developed based on the historical ramping events and ramp forecast errors to directly forecast ramping features (e.g., ramping magnitude, duration, and rate) at different timescales. This paper focuses on the indirect ramp forecasting method, with the aim to identify the benefits of improved wind power forecasting on extreme events. 
Wind forecast models are commonly divided into two categories based on the data utilized [18]. Statistical forecasting is based on the analysis of historical time series of wind; whereas physics-based forecasting uses numerical weather prediction (NWP) models that may include statistical corrections. The first type of forecast model generally provides reasonable results in the estimation of long-term horizons, such as mean monthly, quarterly, and annual wind speed, or for very short timescales, such as a few hours [19]. The impact of atmospheric dynamics becomes more important for short-term horizons of a few hours to day ahead, and NWP models often produce more accurate forecasts on these timescales. Wind forecasts can have different forecasting objectives for multiple customers, including wind power plant owners, utility companies, and independent system operators (ISOs) [20]. For ISOs, the capability to forecast rapid changes in wind power generation is a major concern; it is also the focus of this paper. This paper investigates the impacts of general wind power forecasting improvements on wind power ramp forecasting performance. A ramp extraction methodology is developed to compare the ramp forecasting accuracy of the improved wind power forecasts to the baseline forecasts.

\subsection{Research Motivation and Objectives}

The Wind Forecast Improvement Project (WFIP) was performed to improve short-term wind power forecasts and determine the value of these improvements to grid operators [21]. Large ramps can significantly influence system economics and reliability, upon which power system operators place primary emphasis. This paper evaluates the performance of wind ramp forecasting based on improved short-term wind power forecasting. The analysis is performed for the WFIP southern study region (Fig. 1) by comparing the experimental WFIP forecast to the current short-term wind power forecast (STWPF) at multiple spatial and temporal scales. The WFIP study region in ERCOT includes $8,296 \mathrm{MW}$ of wind capacity (or approximately $85 \%$ of the total rated wind output) spread throughout 84 wind power plants. Short-term forecasts of 1- to 6-hour-ahead (1HA to 6HA) wind power output are analyzed.

The following topics are discussed in the remainder of the paper: (i) the WFIP southern study region and developed improved wind forecasting system; (ii) a statistical analysis to compare the performance of the WFIP to the current STWPF for ERCOT; (iii) the ramp extraction and forecasting methodology and the metrics for assessing ramp forecasting; and (iv) results and discussion of the ramp forecasting as a result of improved wind power forecasts.

\section{Wind Forecast Improvement Project (WFIP)}

WFIP encompassed two study regions: the northern study region and the southern study region [21, 22]. In this paper, we focus on the ramp forecasting performance in the WFIP southern study region.

\subsection{WFIP Southern Study Region}

ERCOT manages the flow of electric power to 24 million Texas customers - representing 85 percent of the state's electric load. The WFIP southern study region covers most of the ERCOT service area, as shown in Fig. 1. As of May 2015, ERCOT had more than 12,000 MW of wind capacity installed and a $40.58 \%$ wind penetration record happened on March 29, 2015 [2]. In this study, 1HA to 6HA wind power forecasts were generated by using both the WFIP and STWPF systems for a nearly 12-month period from October 2011 to mid-September 2012.

\subsection{Wind Forecasting System}

The existing ERCOT's wind power forecasting system, short-term wind power forecast (STWPF), is used as a baseline for this study [22]. The STWPF used Mesoscale Atmospheric Simulations System model forecasts with initial conditions and boundary conditions from the Global Forecast System and the North American Mesoscale Model. Ensemble methods have been shown to produce more accurate forecasts. The WFIP experimental forecast system consists of an ensemble of high-resolution rapid-update NWP models. Each of these ensemble members incorporates a variety of model configurations, physics parameterizations, and data assimilation techniques. The purpose of integrating all of these ensemble members into one system is to construct an optimized composite forecast able to predict forecast uncertainty and assess the relative performance of different modeling approaches. 
Figure 2 shows the overall framework of the wind power forecasting system. The WFIP ensemble members include [22]:

i. The National Oceanic and Atmospheric Administration's 3-km High-Resolution Rapid Refresh model, updated hourly;

ii. Nine NWP models updated every 2 hours on a $5-\mathrm{km}$ grid:

(a) Three configurations of the Advanced Regional Prediction System [23, 24];

(b) Three configurations of the Weather Research and Forecasting model [25];

(c) Three configurations of the Mesoscale Atmospheric Simulations System [26]; and

iii. An Advanced Regional Prediction System model updated every 6 hours on a 2-km grid.

The data from additional sensors deployed for this project, as well as tower data from a set of participating wind power plants within Texas, were assimilated into most of the ensemble members; however, the data from the project sensors were withheld from some ensemble members to gauge their impact on the forecasts [22].

A model output statistics (MOS) procedure was applied to the forecasts from each NWP system. The MOS is designed to correct systematic errors of relevant NWP meteorological variables (e.g., wind speed and direction) at forecast sites. In the WFIP system, a screening multiple linear regression approach was used for each wind power plant site and each NWP model. The purpose of MOS is to remove systematic errors due to unresolved sub-grid processes, limitations in model physics, or data assimilation techniques. There is a lag before NWP forecast data are available due to the time required for data gathering, analysis, initialization, and model execution. Thus, there is typically more recent data available than what is used to initialize the latest available NWP run. There are, however, time series prediction schemes that take advantage of the newer data to improve the NWP forecast performance for the 0 - to 2-hour look-ahead period. In addition to the MOS procedure, the ensemble includes statistical predictions based purely on recent time series of observational data, known as the persistence adjust (PA) method [18]. The PA method determines the initial forecast bias at the time of forecast generation and then applies a static bias correction to the remainder of the 6-hour forecast. The statistical adjustment procedure corrects the initial bias and applies a separate bias correction independently to each forecast interval.

The MOS output for the individual NWP systems was then used as input to an optimized ensemble model, which created a composite deterministic or probabilistic forecast from the set of MOS-adjusted NWP forecasts. For a deterministic forecast, the optimized ensemble model training strategy was based on a rolling sample of the last 30 days to weight each individual forecast according to its performance. The strategy is to assign large weights to forecast members that are likely to perform better based on previous forecasts and observations in the training sample. More details of the forecasting system can be found in [22].

\subsection{Wind Power Forecasting Performance Comparison between WFIP and STWPF}

A comprehensive statistical analysis was performed in Ref. [27] to evaluate the forecast improvements provided by WFIP. Standard statistical metrics (i.e., Pearson's correlation coefficient, root mean square error (RMSE), mean absolute error (MAE), and mean bias error (MBE)) and distributions of wind power forecast errors were used to compare the performance of WFIP and STWPF at different forecast horizons. Results showed that the experimental WFIP provided better results than the current STWPF at all forecast timescales.

To extend the results from Ref. [27] and further illustrate the forecast trend from 1HA to 6HA timescales, Fig. 4 shows the wind power forecast error percentiles (from the 10th to 90th percentile) at different forecast horizons. It is observed in Fig. 4 that the 10th to 90th percentiles of forecast errors continuously decrease with the forecast horizon. A comparison of the STWPF (in Figs. 3(a) and 4(a)) to the WFIP forecasts (in Figs. 3(b) and 4(b)) shows that the $1 \mathrm{HA}$ to $6 \mathrm{HA}$ forecasts from the WFIP performed significantly better than the 1HA to 6HA forecasts from the STWPF.

\section{Ramp Extraction and Forecasting Methodology}


In this paper, we employed four definitions of significant wind power ramps based on the power change magnitude, direction, and duration. The optimized swinging door algorithm (OpSDA) $[28,29]$ was adopted to extract the ramp events from both the actual and forecasted wind power series at different timescales. A suite of metrics was proposed or adopted to evaluate the performance of ramp extraction and forecasting.

Significant wind power ramps can be defined based on the power change magnitude, direction, and duration. The same four definitions proposed in [27] are investigated in this paper:

- Significant Ramp Definition 1 -- the change in wind power output is greater than $30 \%$ of the installed wind capacity without constraining the ramping duration.

- Significant Ramp Definition 2 -- the change in wind power output is greater than $25 \%$ of the installed wind capacity within a time span of 4 hours or less.

- Significant Ramp Definition 3 -- a significant ramp rate is defined as the change rate in wind power output that is greater than $10 \%$ of the installed wind capacity per hour.

- Significant Ramp Definition 4 -- a significant up-ramp is defined as the change in wind power output greater than $20 \%$ of wind capacity within a time span of 4 hours or less; a significant down-ramp is defined as the change in wind power output greater than $15 \%$ of the installed wind power capacity within a time span of 4 hours or less [16].

\subsection{Ramp Extraction Using the Optimized Swinging Door Algorithm (OpSDA)}

Ramps are extracted through a linear piecewise approximation to the original time series of actual or forecasted wind power in this study. The swinging door algorithm (SDA) was adopted previously in [27] to extract wind power ramps. However, the standard SDA did not optimally determine the tunable parameter and optimally segregate the wind power signal. To improve the accuracy of ramps extraction, in this paper we adopt a recently developed ramp extraction method, the optimized SDA (OpSDA) [28, 29]. To determine any significant ramp or ramp rate as defined by definitions 1 to 4 , the start and end points of all ramps in a given time series of wind power need to be identified. Toward this end, the OpSDA was adopted to extract ramp periods in a series of power signals by identifying the start and end points of each ramp. The OpSDA is capable of automatically addressing the changes in ramp definitions in terms of capacity and temporal differences.

The swinging door algorithm (SDA) allows for the consideration of a threshold parameter influencing the algorithm's sensitivity to ramp variations. The OpSDA improves the performance of the original SDA through optimization. First, the SDA is utilized to segregate the wind power generation into consecutive segments in a piecewise linear fashion. Then a dynamic programming approach is used to combine adjacent segments into significant ramps when the decision thresholds are met. An increasing length score function, $\mathrm{S}$, is designed based on the length of the interval segregated by the SDA. The dynamic programming seeks to maximize the length score function, which corresponds to a ramp event. Figure 5 illustrates the overall structure of the OpSDA to identify significant ramps. Based on the four ramp definitions, significant ramps can be identified. Each significant ramp is characterized by the (i) start and end hours; (ii) wind power at the start and end points; and (iii) direction of the ramp. An increasing length score function, $S$, is designed based on the length of the interval segregated by the SDA. The optimization problem seeks to maximize the length score function, which corresponds to a ramp event. Given a time interval, $(i, j)$, of all discrete time points and an objective function, $J$, of the dynamic programming algorithm, a wind power ramping event is detected by maximizing the objective function:

$$
\begin{aligned}
& J(i, j)=\max _{i<k \leq j}[S(i, k)+J(k, j)], i<j \\
& \text { s.t. } \\
& \begin{aligned}
S(i, j) & >S(i, k)+S(k+1, j), \forall i<k<j \\
& S(i, j)=(j-i)^{2} \times R(i, j)
\end{aligned}
\end{aligned}
$$

where $J(i, j)$ can be computed as the maximum over $(j-i)$ subproblems. The term of $S(i, k)$ is a positive score value corresponding to the interval, $(i, k)$, which conforms to a super-additivity property in Eq. (2). There is a family of 
score functions satisfying Eq. (2), and the score function expressed in Eq. (3) is adopted in this research. $R(i, j)$ represents a ramp within the time interval $(i, j)$. A detailed description of the OpSDA can be found in $[28,29]$.

\subsection{Metrics for Evaluating Significant Ramp Forecasting Performance}

A suite of ramp event detection metrics is used to evaluate the performance of ramp forecasting, including a contingency table, categorical statistics, and performance diagrams. Categorical statistics provide measures of accuracy and skill for forecasts of notable events, such as ramps in power, detrimental temperatures, or rainfall. The adopted metrics include the probability of detection (POD), the critical success index (CSI), the frequency bias score (FBIAS), and the success ratio (SR) $[12,27]$. They are calculated based on a contingency table in Table 1 that provides a measure of skill for the forecast ramps approaching the actual ramps. True positive (TP) represents the number of forecasted ramps (forecast YES) that are actually observed in the actual power output (observed YES); false positive (FP) is the number of forecasted ramps that are not observed in the actual wind power (observed NO); false negative (FN) represents the number of observed ramps (observed YES) that are not predicted by the wind forecasting system (forecast $\mathrm{NO}$ ); true negative (TN) is the number of non-occurring events for both observed and forecast results; and $\mathrm{N}$ is the total number of events. The relationship among the POD, CSI, FBIAS, and FAR can be visualized on a performance diagram [30].

Categorical statistics provide measures of accuracy and skill for forecasts of notable events, such as ramps in power, detrimental temperatures, or rainfall. Based on the contingency table, a suite of metrics can be derived for ramp forecasting performance evaluation, given as follows.

Probability of detection (POD) is defined as the ratio between the number of true positives and the number of observed positives, which indicates the fraction of observed YES events that are actually forecasted.

$$
P O D=\frac{T P}{T P+F N}
$$

Critical success index (CSI) is used to measure the fraction of observed and/or forecasted events that are correctly predicted, given by

$$
C S I=\frac{T P}{T P+F N+F P}
$$

The value of CSI is between 0 and 1, with 1 representing perfect prediction. The CSI considers only observed and forecasted ramps, excluding true negative events.

Frequency bias score (FBIAS) measures the ratio of the frequency of forecasted YES events to the frequency of observed YES events.

$$
F B I A S=\frac{T P+F P}{T P+F N}
$$

The ramp forecast system tends to underforecast when FBIAS $<1$, and it tends to overforecast when FBIAS $>1$.

False alarm ratio (FAR) measures the fraction of predicted YES events that did not occur, given by

$$
F A R=\frac{F P}{F P+T P}
$$

The metric success ratio (SR) is calculated from FAR by subtracting it from 1 . SR measures the fraction of predicted YES events that occurred.

The relationship among the POD, CSI, FBIAS, and FAR can be visualized on a performance diagram [31] based on

$$
C S I=\frac{1}{\frac{1}{P O D}+\frac{1}{1-F A R}-1}
$$




\section{Evaluating Significant Ramp Forecasting Performance Based on Improved Wind Power Forecasts}

273

274

275

276

277

278

279

280

281

282

283

284

285

The performance of wind power ramp forecasting is affected by many factors such as the ramp extraction algorithm, wind power forecasts, wind penetration level in the power system, and others. In this paper, we focus on analyzing the effects of the wind power forecast improvements on the performance of ramp forecasting. The true position forecast defined by Greaves et al. [13] is adopted and modified in this study. Greaves et al. [13] defined a true positive forecast as a forecast ramp with a measured ramp of the same direction (either up or down) within \pm 12 hours of the time of the forecast ramp. In this study, a true positive forecast is defined as a forecast ramp with a measured ramp of the same direction within \pm 6 hours of the time of the forecast ramp. This \pm 6 -hours range could provide sufficient data for temporal uncertainty analysis and maintain a realistic connection between forecast and measured significant wind ramp events. We have analyzed the ramp forecasting performance at different spatial and temporal scales. The annual, seasonal, and monthly ramp forecasting evaluation results are analyzed and discussed in the following sections. In this paper, the notation "annual" represents the nearly 12-month period from October 2011 to mid-September 2012.

\subsection{Annual Ramp Forecasting Performance}

Table 2 lists the number of significant up and down ramps in actual wind power generation throughout the whole year for all wind farms. It is seen that there are generally more up ramps than down ramps according to four significant ramp definitions. By comparing the four definitions, there are more ramps fitting to Ramp Definition 4 than to other definitions.

A performance diagram (as illustrated in Fig. 6) is used for quantitative and visual analysis, thereby understanding whether the wind power ramp forecasting performance is improved. Figure 6 illustrates the annual ramp forecasting performance of STWPF and WFIP for different forecast timescales and significant ramp definitions. In the performance diagrams, (i) the left axis represents the value of POD; (ii) the bottom axis represents the success ratio; (iii) the diagonal dashed lines represent FBIAS with the values shown on the right and top axes; and (iv) the solid curves show CSI with the values on the right-inside graph border. The figure shows the 4HA, $5 \mathrm{HA}$, and $6 \mathrm{HA}$ forecasts. The performance diagram shows the ramp forecast performance space - that is, the overall ramp forecast metrics are improved as the forecast moves toward the upper right of the diagram.

The performance diagram in Fig. 6 compares the annual ramp forecasting performance of the STWPF to the WFIP for different forecast timescales and ramp definitions. Three forecasting timescales are evaluated: 4HA, 5HA, and 6HA. It is observed in Fig. 6(a) according to Ramp Definition 1 that: (i) the 4HA WFIP has a larger success ratio, POD, and CSI values than the 4HA STWPF; (ii) the 5HA WFIP also has a larger success ratio, however a smaller POD, than the 5HA STWPF; and (iii) the 6HA WFIP has a larger success ratio and CSI values than the 6HA STWPF. Similar results are also observed according to the Ramp Definition 2 as shown in Fig. 6(b). With Ramp Definitions 3 and 4, WFIP performs better than STWFP at all forecast timescales. Overall, the ramp forecasting as a result of the improved wind power forecasts has been improved. The FBIAS values are smaller than one for all cases, which indicates that both the WFIP and STWPF ramp forecasts tend to underforecast. This underforecasting trend could alert the power system operators to employ appropriate strategies to compensate for wind power ramps, such as holding more reserves.

Figure 7 shows the distribution of the ramp magnitude. For ramp Definitions 1 and 2, at the peak of the distribution, both WFIP and STWFP present larger ramp magnitude comparing to the actual wind power ramps. For Definition 3 in Fig. 7(c), the ramp magnitude distribution presents two peaks in the actual ramps, which are also successfully forecasted by both WFIP and STWPF.

\subsection{Ramp Forecasting Performance at Multiple Spatial Scales}


Understanding the ramp forecasting performance within different spatial scales can provide a better understanding of the flexibility requirements and reliability impacts of wind integration on the grid. Four scenarios are analyzed based on the number of wind power plants, including: (i) all wind power plants within the WFIP southern study region with a 8,296-MW capacity; (ii) 41 wind power plants with an aggregated 4,863-MW capacity; (iii) 4 wind power plants with an aggregated 1,073-MW capacity; and (iv) a single wind power plant with an approximate 500MW capacity. Figure 8 compares WFIP ramp forecasting performance at multiple spatial and temporal scales with different significant ramp definitions. It is observed that, for Definition 3, the success ratio decreases with aggregating more wind power plants, indicating that there are more predicted YES events that actually occurred for a small wind power capacity. For Definitions 2 and 3, as shown in Figs. 8(b) and (c), respectively, the ramp forecasting for the single wind power plant also presents relatively larger POD values, indicating that there are more observed YES events that are actually forecasted. However, the ramp forecasting with more aggregated wind power plants (all plants and 41 plant scenarios) presents relatively larger POD and success ratio values for Definition 1, as shown in Fig. 8(a). It is shown that the performance of ramp forecasting is sensitive to the significant ramp definition. For power system operations, it is important to make multiple strategies to handle different types of wind power ramps based on the definition of significant ramps. Table 3 lists the number of significant ramps for different spatial aggregations with different significant ramp definitions. It is seen that the number of significant ramps is decreasing by aggregating more wind power plants, which shows the smoothing effect from geographic diversity in wind power ramps.

Figure 9 shows the distribution of the ramp magnitude for different spatial aggregations. For all the four ramp definitions, the distribution peak of the single site case has a relatively smaller probability density than multiple sites cases. This observation is more evident with the ramp Definition 3 in Fig. 9(c). For all spatial aggregations, at the peak of the distribution, both WFIP and STWFP present larger ramp magnitude comparing to the actual wind power ramps.

\subsection{Seasonal Ramp Forecasting Performance}

In addition to the annual ramp forecasting comparison, the seasonal ramp forecasting performance is also compared between different forecasting timescales for all wind power plants, as illustrated in Fig. 10. It is seen from all the four significant ramp definitions in Figs. 10(a)-(d) that, the ramp forecasting performs relatively better in fall and worse in summer for both the WFIP and STWPF. This can be partially attributed to the features responsible for ramps. The summer tends to be more convective (mesoscale); hence, it is more challenging to forecast (especially on the 4HA timescale) than the larger synoptic scale (such as fronts) features that cause ramps in the colder seasons. It is observed in Fig. 10(a) that during summer the ramp forecasting based on WFIP performs significantly better than that based on STWPF. However, during fall the ramp forecasting based on STWPF performs slightly better. This significant improvement of ramp forecasting performance in summer based on the improved wind forecasts could play an important part in enhancing system economics and reliability, considering that high electric demand is generally expected in ERCOT during the summer period. After comparing the ramp forecasting performances among the four different significant ramp definitions, it is evident that the WFIP ramp forecasts generally have a larger success ratio, POD, and CSI values with Definition 4, as shown in Fig. 10(d). The FBIAS value is also closer to one with Definition 4.

Figure 11 shows the distribution of the ramp magnitude for different seasons. It is seen that for all four seasons, at the peak of the distribution, both WFIP and STWFP present larger ramp magnitude comparing to the actual wind power ramps. It is also observed that distribution curves of the summer case are more fluctuated compared to other season. This again illustrates that ramp forecasting is relatively more challenging in summer.

\subsection{Monthly Ramp Forecasting Performance}

Figure 12 compares monthly ramp forecasting performance among the 4HA to 6HA STWPF and WFIP forecasts for all wind power plants within the WFIP southern study region. The 12 points represented by each symbol (e.g., circle) indicate the ramp forecasting performance in each month. It is observed that with different 
forecasting timescales and months, the variation in the ramp forecasting performance based on the significant ramp Definitions 2 and 4 is relatively less than that based on the ramp Definitions 1 and 3. Although the annual ramp forecasting tends to underforecast (FBIAS $<1$ as shown in Fig. 6), the system tends to overforecast in a few months as shown in Fig. 12 in the cases of FBIAS $>1$.

Figure 13 shows the distribution of the ramp magnitude at four typical months (i.e., March, June, September, and December). It is seen that distribution curves in September and December case are more fluctuated, especially at the right tail of the distribution with Ramp Definition 3. We also found that most of the WFIP and STWPF distribution peaks are on the left side of the actual distribution peak, which again validate the underforecasting tendency.

\subsection{Results Discussion}

The annual, seasonal, and monthly ramp forecasting evaluation results were analyzed. Overall, the ramp forecasting was improved as a result of the improved wind power forecasts. It was found from the seasonal analysis that, the ramp forecasting performed relatively better in fall and worse in summer for both the WFIP and STWPF. This could be partially attributed to the features responsible for ramps. The summer tends to be more convective (mesoscale); hence, it is more challenging to forecast (especially on the 4HA timescale) than the larger synoptic scale (such as fronts) features that cause ramps in the colder seasons. We found from the multiple spatial scale analysis that, the number of significant ramps decreased when aggregating more wind power plants, which showed the smoothing effect from geographic diversity in wind power ramps. By comparing the four significant ramp definitions, we found that the performance of ramp forecasting was highly sensitive to the significant ramp definition. Therefore, it is important for power system operators to make multiple strategies to handle different types of wind power ramps based on their system characteristics, such as the flexibility capability, wind penetration level, etc.

\section{Conclusion}

This paper characterized the ramp forecasting performance of two forecasting systems: the experimental forecasts from the Wind Forecast Improvement Project (WFIP) and the current Electric Reliability Council of Texas (ERCOT) short-term wind power forecast (STWPF). The WFIP experimental forecast system consists of an ensemble of high-resolution rapid-update numerical weather prediction (NWP) models in conjunction with a model output statistics process. A suite of statistical metrics were used to evaluate the overall improvement of the WFIP in short-term wind power forecasting accuracy for different forecasting horizons. Statistical analyses of the results showed that in most seasons and months the experimental WFIP provided better performance than the current STWPF for all forecasting horizons.

An evaluation of the ramp forecasting improvement was performed based on four types of significant ramp definitions, which were determined based on the power change magnitude, direction, and duration. The wind power ramps were extracted using the optimized swinging door algorithm (OpSDA). Results showed that the OpSDA successfully captured wind power ramps for different definitions in terms of capacity and temporal differences. The ramp extraction results also found that the ramp magnitude varied more when the duration of the ramps was relatively short.

The ramp forecasting comparison results showed that enhanced WFIP forecasts improved the accuracy of wind power ramp forecasting, especially during the summer period in ERCOT. The results also showed that the ramp forecasting for both the WFIP and STWPF tended to underforecast during the whole year. The ramp forecasting performed relatively better with the ramp definition based on all three attributes: the power change direction, magnitude, and duration of the ramps.

Deterministic ramp forecasts are not able to predict the likelihood of occurrence or likelihood of different ramp event scenarios. An alternative to deterministic ramp forecasts is probabilistic ramp forecasts, which could provide a probability distribution of ramps or ramp rates. Future work will (i) develop probabilistic wind power ramp forecasts through NWP ensembles and (ii) evaluate the impacts of improved short-term wind power forecasting on probabilistic ramp forecasts. 


\section{Acknowledgments}

This work was supported by the National Renewable Energy Laboratory under Subcontract No. XGJ-6-62183-01 (under the U.S. Department of Energy Prime Contract No. DE-AC36-08GO28308).

\section{References}

[1] EIA, "Electric Power Monthly," U.S. Department of Energy2015.

[2] ERCOT. Electric Reliability Council of Texas. Available: http://www.ercot.com/

[3] XcelEnergy. (2015). Available: https://www.xcelenergy.com/

[4] D. Lew, G. Brinkman, E. Ibanez, B. Hodge, and J. King, "The western wind and solar integration study phase 2," Contract, vol. 303, pp. 275-3000, 2013.

[5] J. Wang, A. Botterud, R. Bessa, H. Keko, L. Carvalho, D. Issicaba, et al., "Wind power forecasting uncertainty and unit commitment," Applied Energy, vol. 88, pp. 4014-4023, 2011.

[6] S. Alessandrini, S. Sperati, and P. Pinson, "A comparison between the ECMWF and COSMO Ensemble Prediction Systems applied to short-term wind power forecasting on real data," Applied Energy, vol. 107, pp. 271-280, 2013.

[7] ERCOT, "Report on the Capacity, Demand, and Reserves in the ERCOT Region, 2015-2024," Electric Reliability Council of Texas, 2014.

[8] E. Ela and B. Kirby, ERCOT event on February 26, 2008: lessons learned: National Renewable Energy Laboratory, 2008.

[9] CAISO, "What the duck curve tells us about managing a green grid," California Independent System Operator, 2013.

[10] T. Mai, R. Wiser, D. Sandor, G. Brinkman, G. Heath, P. Denholm, et al., "Exploration of High-Penetration Renewable Electricity Futures. Vol. 1 of Renewable Electricity Futures Study," National Renewable Energy Laboratory, Golden, CO, Tech. Rep. NREL/TP-6A20-52409-1, 2012.

[11] C. Gallego-Castillo, A. Cuerva-Tejero, and O. Lopez-Garcia, "A review on the recent history of wind power ramp forecasting," Renewable and Sustainable Energy Reviews, vol. 52, pp. 1148-1157, 2015.

[12] C. Ferreira, J. Gama, L. Matias, A. Botterud, and J. Wang, "A survey on wind power ramp forecasting," Argonne National Laboratory (ANL), 2011.

[13] B. Greaves, J. Collins, J. Parkes, and A. Tindal, "Temporal forecast uncertainty for ramp events," Wind Engineering, vol. 33, pp. 309-319, 2009.

[14] H. Zheng and A. Kusiak, "Prediction of wind farm power ramp rates: A data-mining approach," Journal of solar energy engineering, vol. 131, p. 031011, 2009.

[15] C. W. Potter, E. Grimit, and B. Nijssen, "Potential benefits of a dedicated probabilistic rapid ramp event forecast tool," in Power Systems Conference and Exposition, 2009. PSCE'09. IEEE/PES, 2009, pp. 1-5.

[16] A. Truewind, "AWS Truewind's final report for the Alberta forecasting pilot project," Wind Power Forecasting PILOT Project, 2008.

[17] J. Freedman, M. Markus, and R. Penc, "Analysis of West Texas wind plant ramp-up and ramp-down events," AWS Truewind, LLC, 2008.

[18] A. M. Foley, P. G. Leahy, A. Marvuglia, and E. J. McKeogh, "Current methods and advances in forecasting of wind power generation," Renewable Energy, vol. 37, pp. 1-8, 2012.

[19] C. Monteiro, R. Bessa, V. Miranda, A. Botterud, J. Wang, and G. Conzelmann, "Wind power forecasting: state-of-the-art 2009," Argonne National Laboratory (ANL), 2009.

[20] A. Botterud, J. Wang, V. Miranda, and R. J. Bessa, "Wind power forecasting in US electricity markets," The Electricity Journal, vol. 23, pp. 71-82, 2010.

[21] J. Wilczak, M. Marquis, C. Finley, and J. Freedman, "The wind forecast improvement project (WFIP): A public/private partnership for improving short term wind energy forecasts and quantifying the benefits of utility operations," US Department of Energy (DOE), Washington, DC, Tech. Rep. DE-EE0003080, 2014.

[22] J. M. Freedman, J. Manobianco, J. Schroeder, B. Ancell, K. Brewster, S. Basu, et al., "The Wind Forecast Improvement Project (WFIP): A Public/Private Partnership for Improving Short Term Wind Energy Forecasts and Quantifying the Benefits of Utility Operations. The Southern Study Area, Final Report," AWS Truepower, LLC, Albany, NY (United States), 2014. 
[23] M. Xue, K. K. Droegemeier, and V. Wong, "The Advanced Regional Prediction System (ARPS)-A multi-scale nonhydrostatic atmospheric simulation and prediction model. Part I: Model dynamics and verification," Meteorology and atmospheric physics, vol. 75, pp. 161-193, 2000.

[24] M. Xue, K. K. Droegemeier, V. Wong, A. Shapiro, K. Brewster, F. Carr, et al., "The Advanced Regional Prediction System (ARPS)-A multi-scale nonhydrostatic atmospheric simulation and prediction tool. Part II: Model physics and applications," Meteorology and atmospheric physics, vol. 76, pp. 143-165, 2001.

[25] W. C. Skamarock, J. B. Klemp, J. Dudhia, D. O. Gill, D. M. Barker, W. Wang, et al., "A description of the advanced research WRF version 2," DTIC Document, 2005.

[26] J. Manobianco, J. W. Zack, and G. E. Taylor, "Workstation-based real-time mesoscale modeling designed for weather support to operations at the Kennedy Space Center and Cape Canaveral Air Station," 1996.

[27] J. Zhang, A. Florita, B.-M. Hodge, and J. Freedman, "Ramp Forecasting Performance From Improved ShortTerm Wind Power Forecasting," in ASME 2014 International Design Engineering Technical Conferences and Computers and Information in Engineering Conference, 2014.

[28] M. Cui, J. Zhang, A. R. Florita, B.-M. Hodge, D. Ke, and Y. Sun, "An Optimized Swinging Door Algorithm for Wind Power Ramp Event Detection," in IEEE Power \& Energy Society General Meeting, Denver, CO, 2015.

[29] M. Cui, J. Zhang, A. R. Florita, B.-M. Hodge, D. Ke, and Y. Sun, "An Optimized Swinging Door Algorithm for Identifying Wind Ramping Events," IEEE Transactions on Sustainable Energy, vol. 7, pp. 150-162, 2016.

[30] P. J. Roebber, "Visualizing multiple measures of forecast quality," Weather and Forecasting, vol. 24, pp. 601608, 2009. 
Table 1. Contingency table for ramp event observation and forecast

\begin{tabular}{|c|c|c|c|}
\hline & Observed YES & Observed NO & Total \\
\hline Forecast YES & $T P$ (hits) & $F P$ (false alarm) & $T P+F P$ \\
\hline Forecast NO & $F N$ (misses) & $T N$ & $F N+T N$ \\
\hline Total & $T P+F N$ & $F P+T N$ & $N=T P+F P+F N+T N$ \\
\hline
\end{tabular}

484

485

486

487

Table 2. Number of observed ramps in the actual wind power for all wind farms

\begin{tabular}{|c|c|c|c|c|}
\hline Ramp Type & Ramp Def. 1 & Ramp Def. 2 & Ramp Def. 3 & Ramp Def. 4 \\
\hline Up Ramps & 187 & 159 & 208 & 245 \\
\hline Down Ramps & 160 & 126 & 150 & 335 \\
\hline
\end{tabular}

Table 3. Number of ramps for different spatial aggregations with different ramp definitions

\begin{tabular}{|c|c|c|c|c|c|}
\hline $\begin{array}{c}\text { Forecasting } \\
\text { Timescale }\end{array}$ & $\begin{array}{c}\text { Spatial } \\
\text { Aggregation }\end{array}$ & Ramp Def. 1 & Ramp Def. 2 & Ramp Def. 3 & Ramp Def. 4 \\
\hline \multirow{4}{*}{4 HA } & 1 site & 635 & 1049 & 1105 & 853 \\
\cline { 2 - 6 } & 4 sites & 514 & 854 & 634 & 684 \\
\cline { 2 - 6 } & 41 sites & 441 & 689 & 305 & 545 \\
\cline { 2 - 6 } & All sites & 436 & 607 & 229 & 474 \\
\hline \multirow{4}{*}{ 5HA } & 1 site & 625 & 1007 & 1098 & 836 \\
\cline { 2 - 6 } & 4 sites & 515 & 815 & 669 & 667 \\
\cline { 2 - 6 } & 41 sites & 448 & 657 & 323 & 526 \\
\hline \multirow{4}{*}{6 HA } & All sites & 427 & 584 & 267 & 450 \\
\cline { 2 - 6 } & 4 site & 615 & 992 & 1038 & 819 \\
\cline { 2 - 6 } & 4 sites & 525 & 817 & 654 & 658 \\
\cline { 2 - 6 } & 41 sites & 441 & 687 & 344 & 556 \\
\hline
\end{tabular}

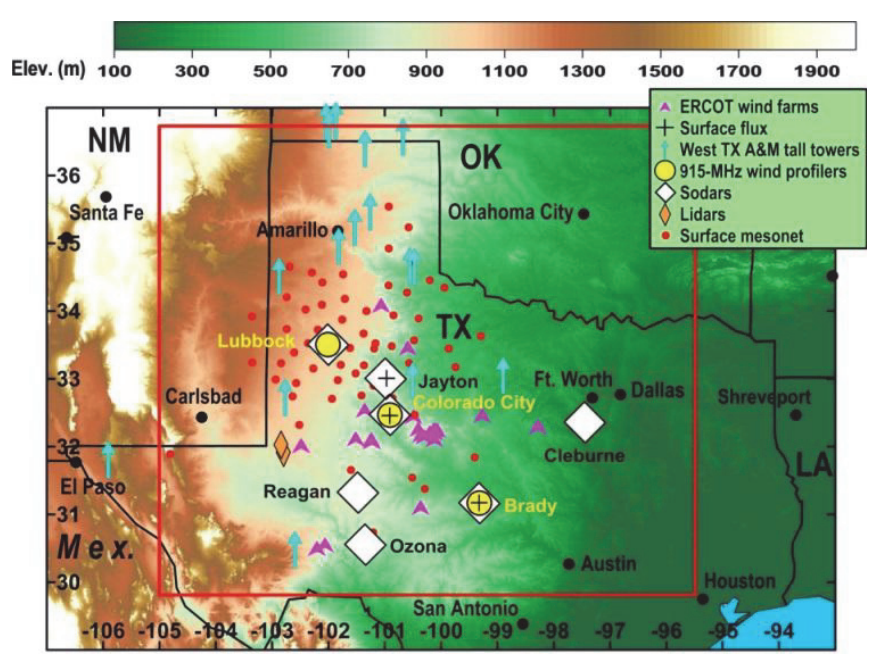

Figure 1. WFIP Southern study region in ERCOT [22] 


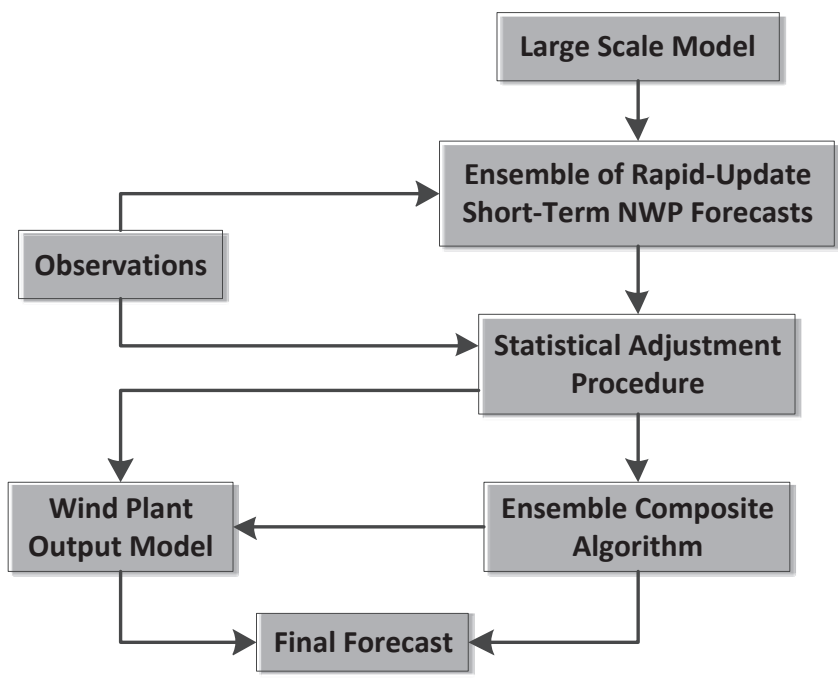

Figure 2. Overall framework of the wind power forecasting system [22]

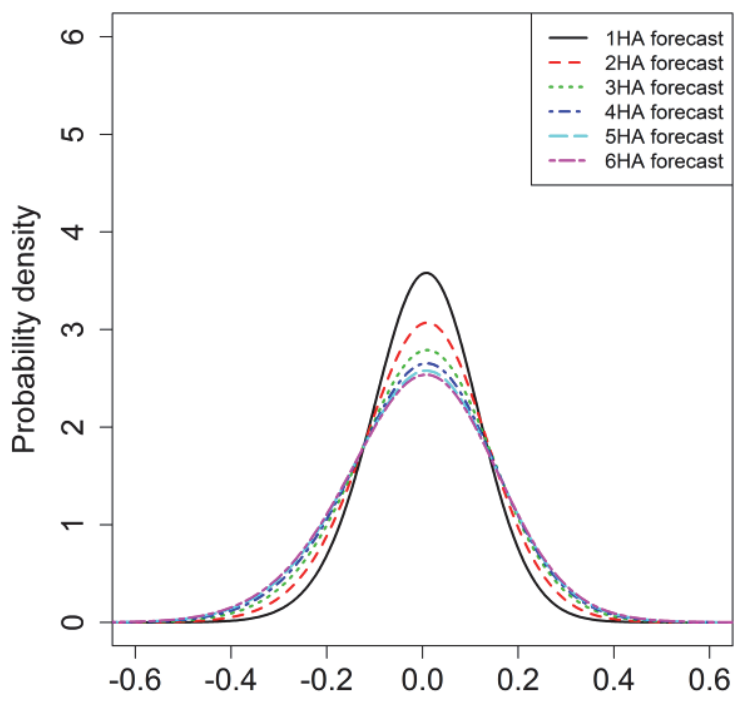

Normalized wind forecast error

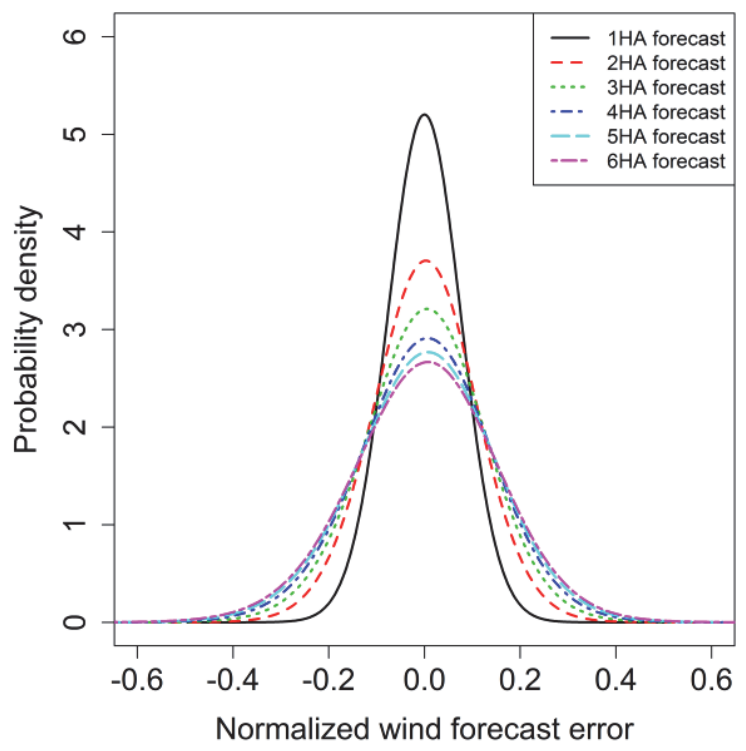

(b) WFIP

(a) STWFP

Figure 3. Distributions of wind power forecast errors at forecast horizons 


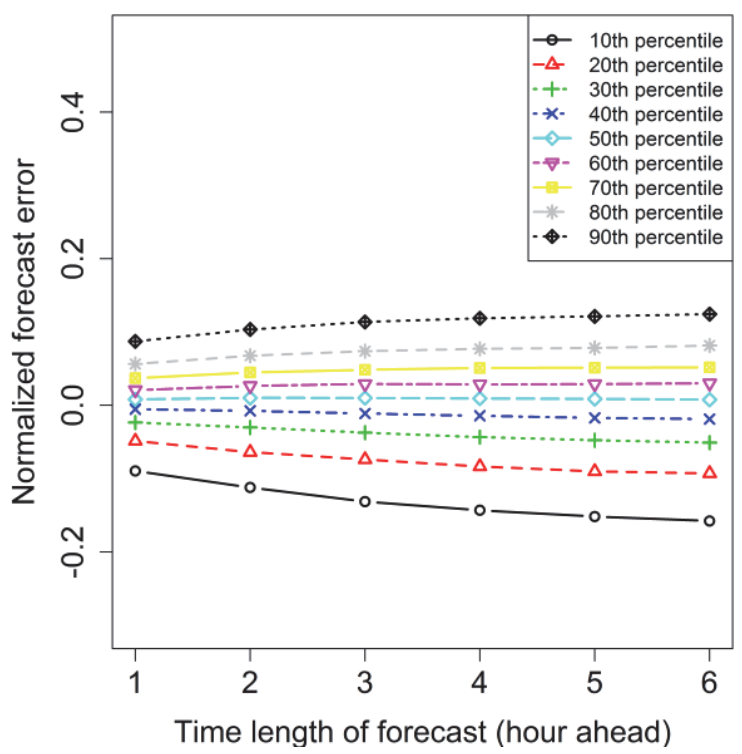

(a) STWFP

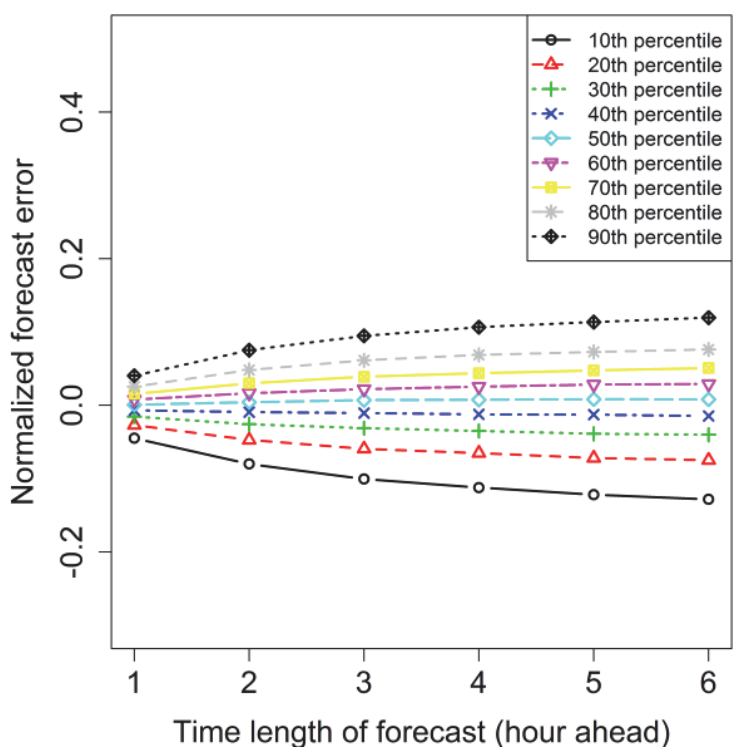

(b) WFIP

Figure 4. 10th to 90th percentiles of wind power forecasts at different forecast horizons

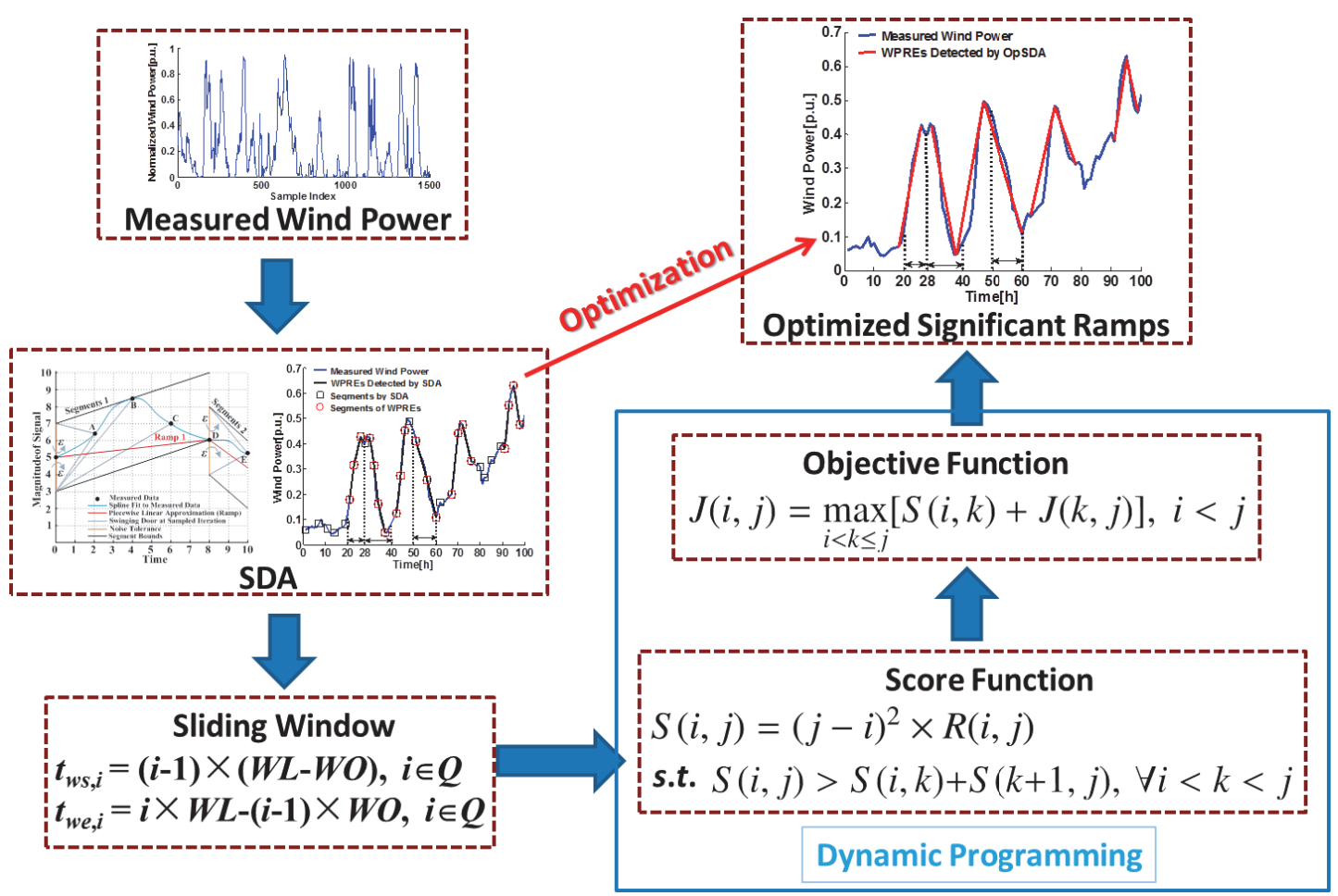

Figure 5. The OpSDA for the extraction of ramps in power from the time series 


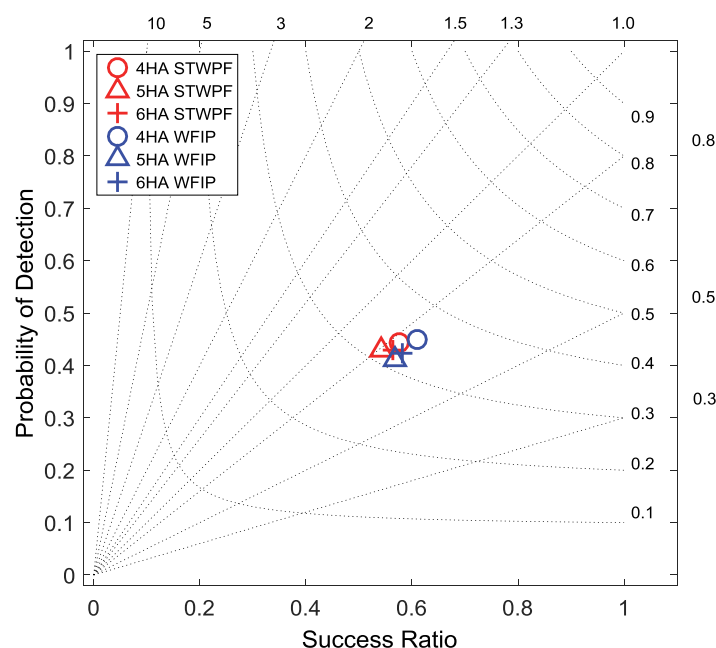

505

(a) Ramp Definition 1: Ramp magnitude only

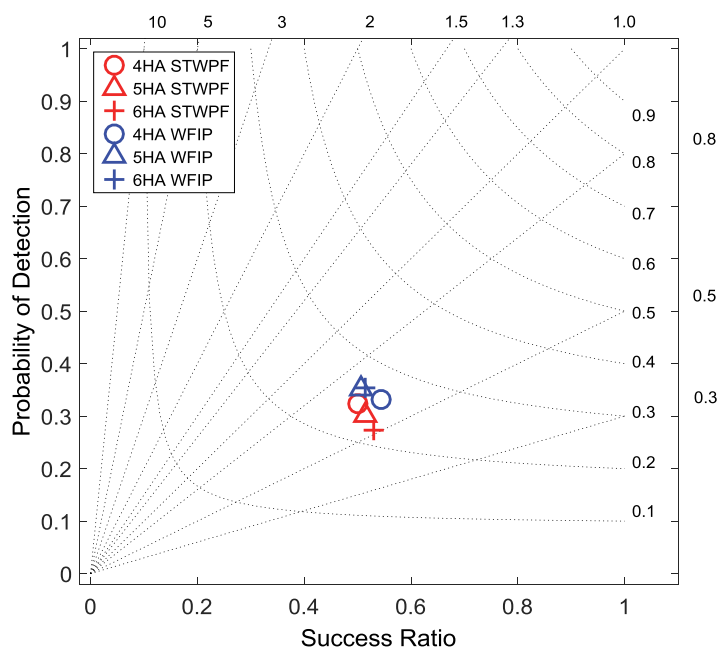

(c) Ramp Definition 3: Ramp rate

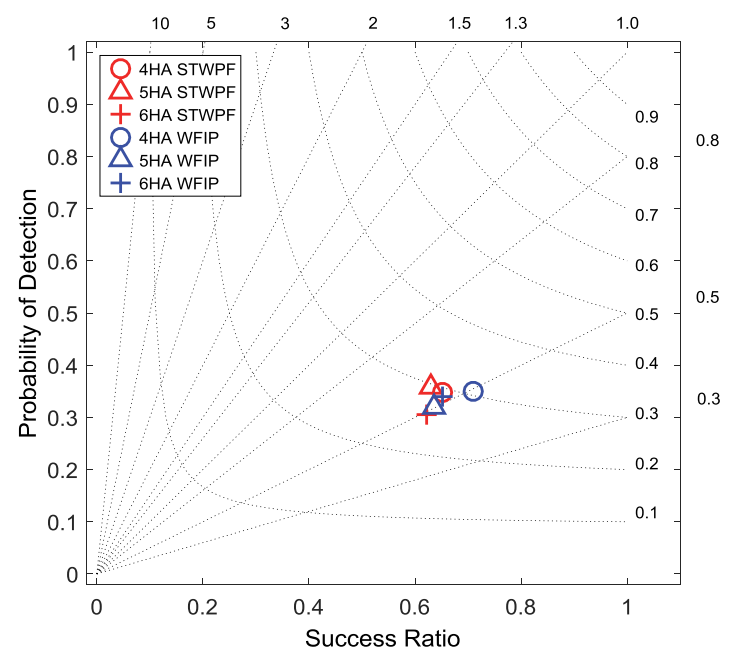

(b) Ramp Definition 2: Ramp magnitude and duration

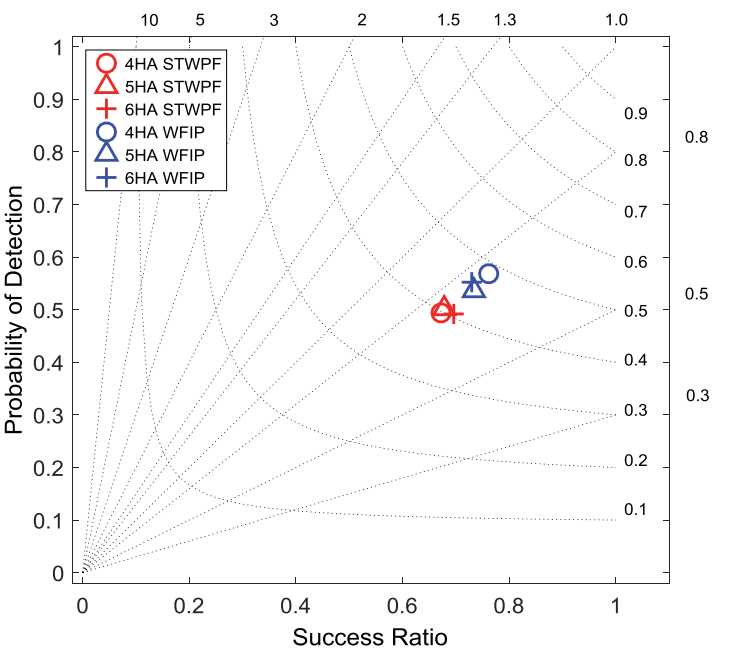

(d) Ramp Definition 4: Ramp direction, magnitude, and duration Figure 6. Comparison of annual ramp forecasting performance of STWPF to WFIP for different forecast timescales and ramp definitions 

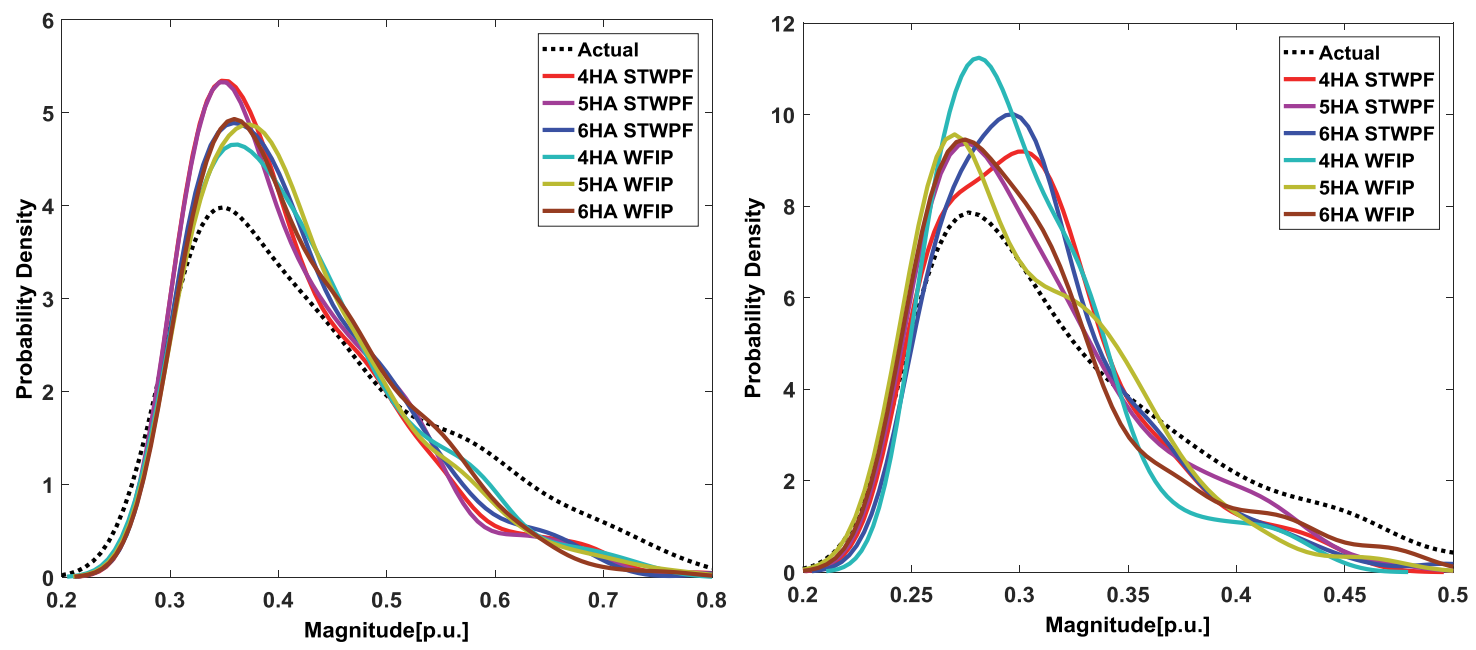

511 (a) Ramp Definition 1: Ramp magnitude only

(b) Ramp Definition 2: Ramp magnitude and duration
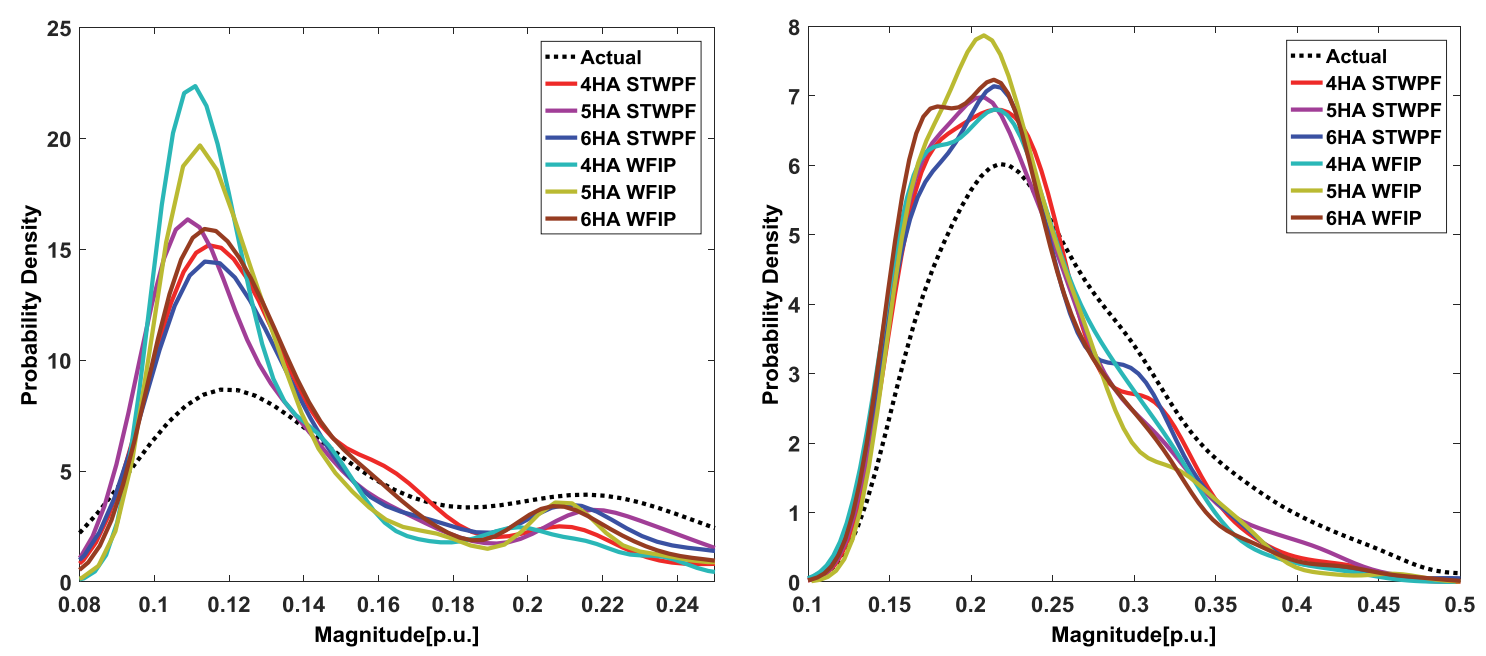

513

(c) Ramp Definition 3: Ramp rate

(d) Ramp Definition 4: Ramp direction, magnitude, and duration

Figure 7. Comparing annual distribution of ramp magnitude between STWPF to WFIP 
516

517

518

519

520

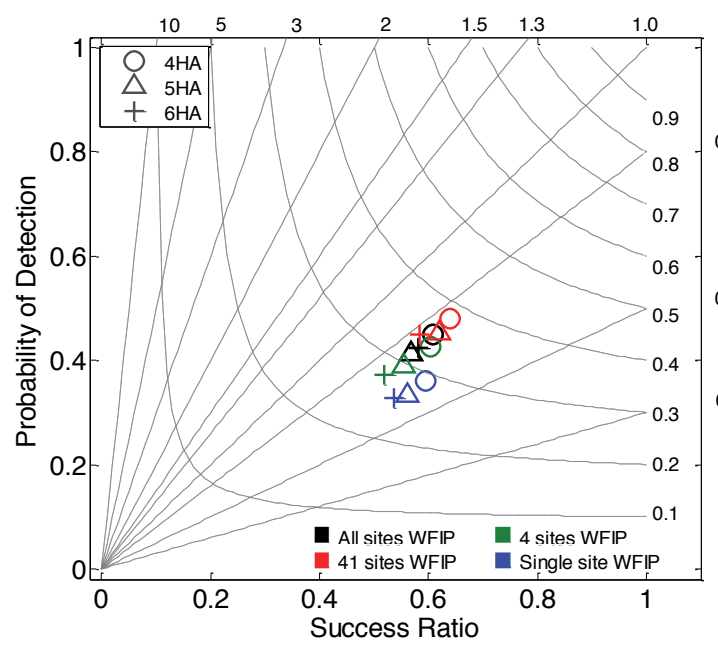

(a) Ramp Definition 1: Ramp magnitude only

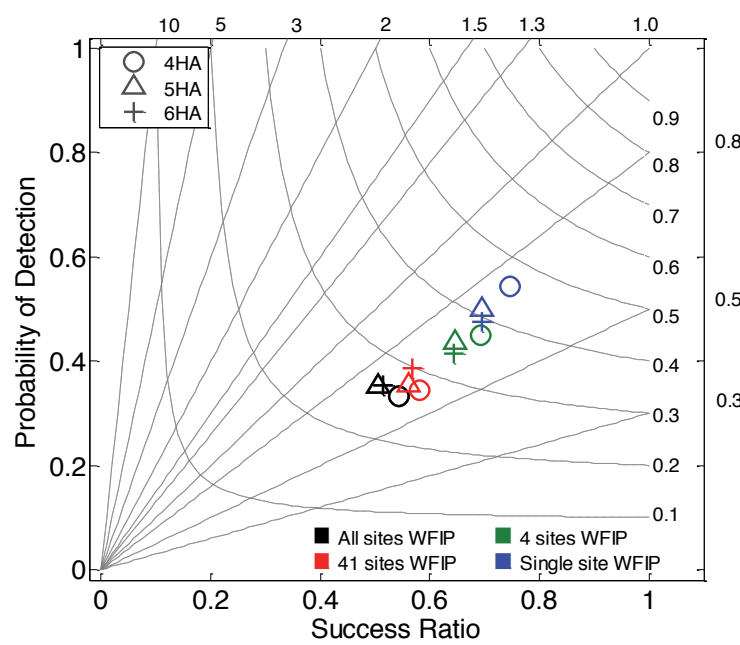

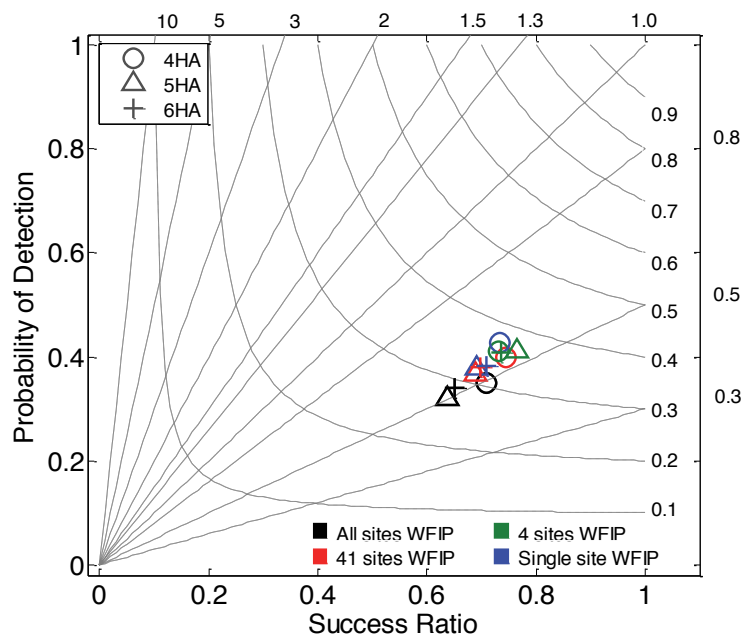

(b) Ramp Definition 2: Ramp magnitude and duration

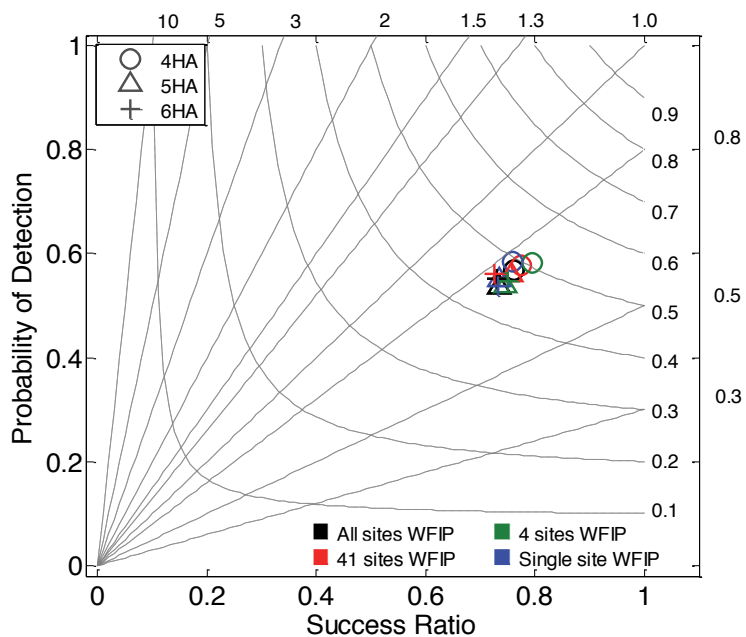

(c) Ramp Definition 3: Ramp rate $\quad$ (d) Ramp Definition 4: Ramp direction, magnitude, and duration Figure 8. Comparison of WFIP ramp forecasting performance for different spatial aggregations 

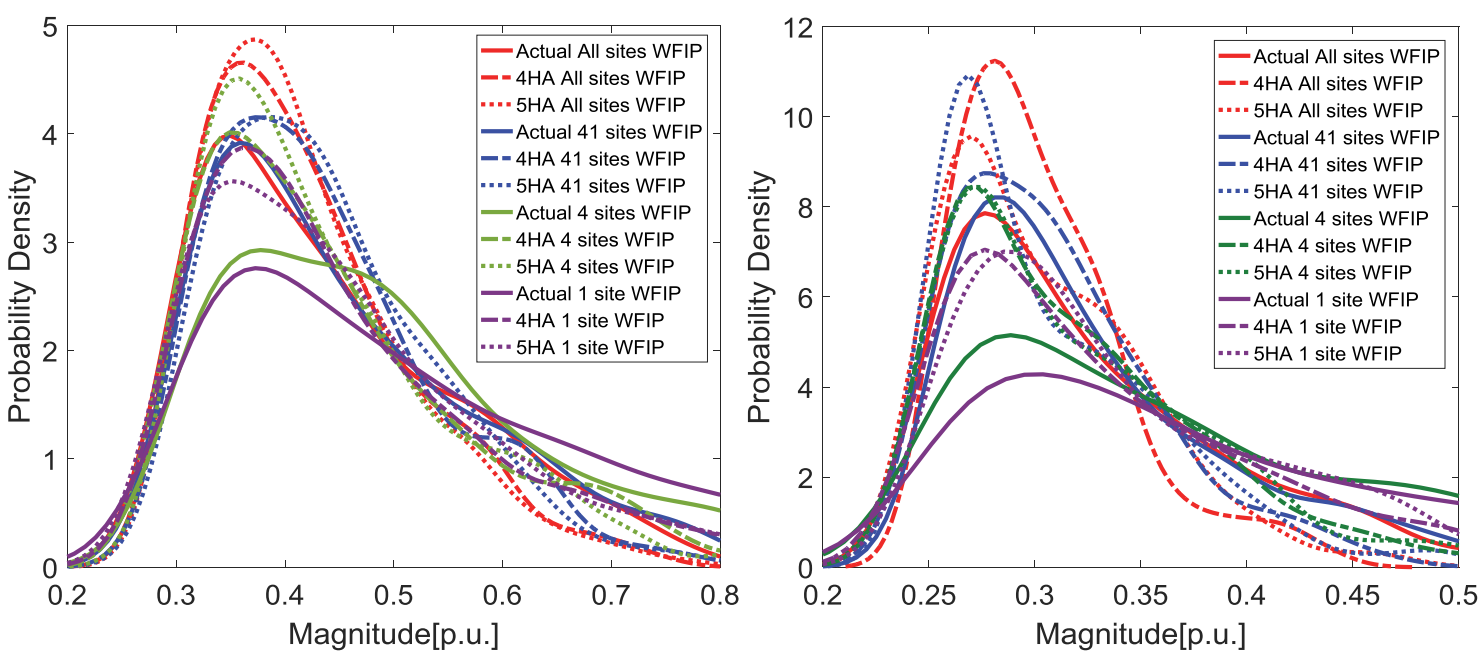

$523 \quad$ (a) Ramp Definition 1: Ramp magnitude only

(b) Ramp Definition 2: Ramp magnitude and duration
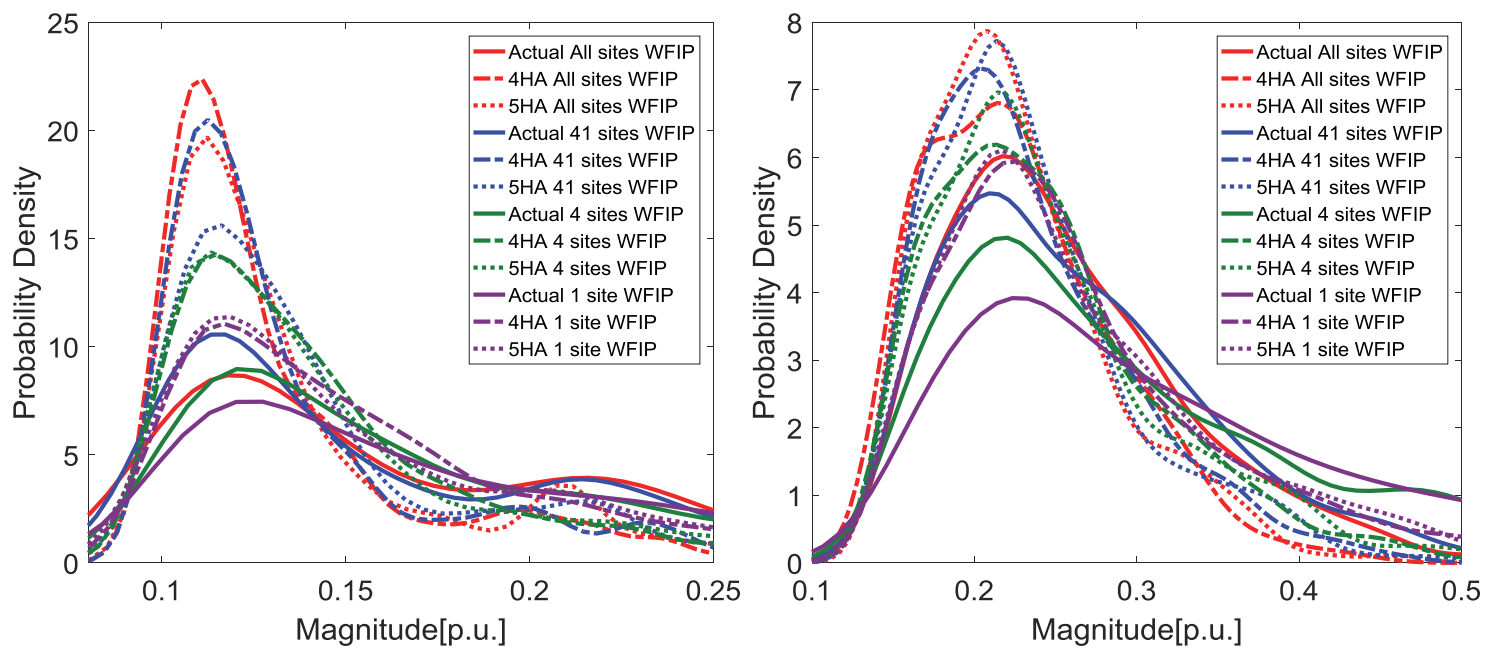

(c) Ramp Definition 3: Ramp rate (d) Ramp Definition 4: Ramp direction, magnitude, and duration Figure 9. Comparing WFIP distribution of ramp magnitude for different spatial aggregations 


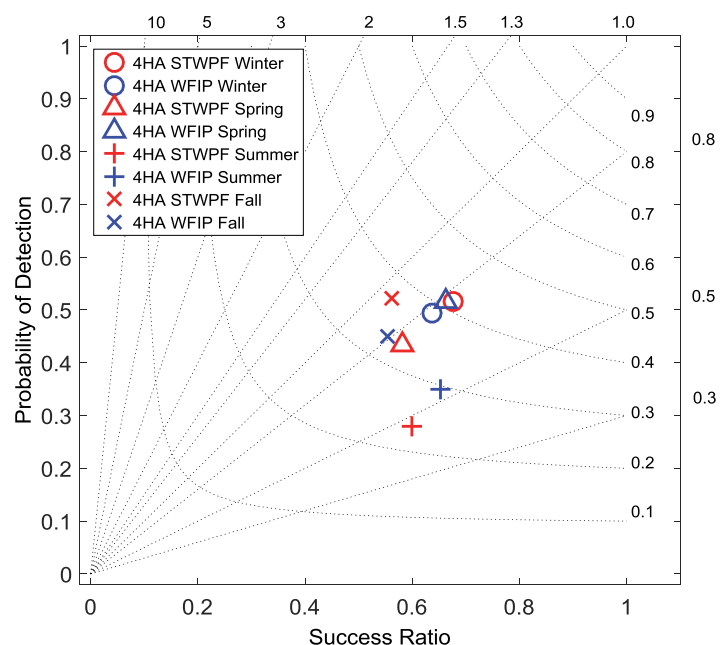

(a) Ramp Definition 1: Ramp magnitude only

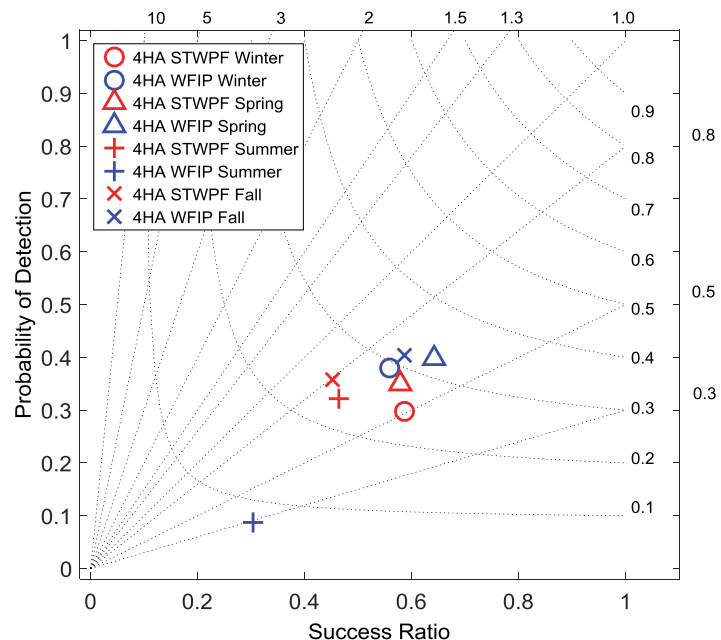

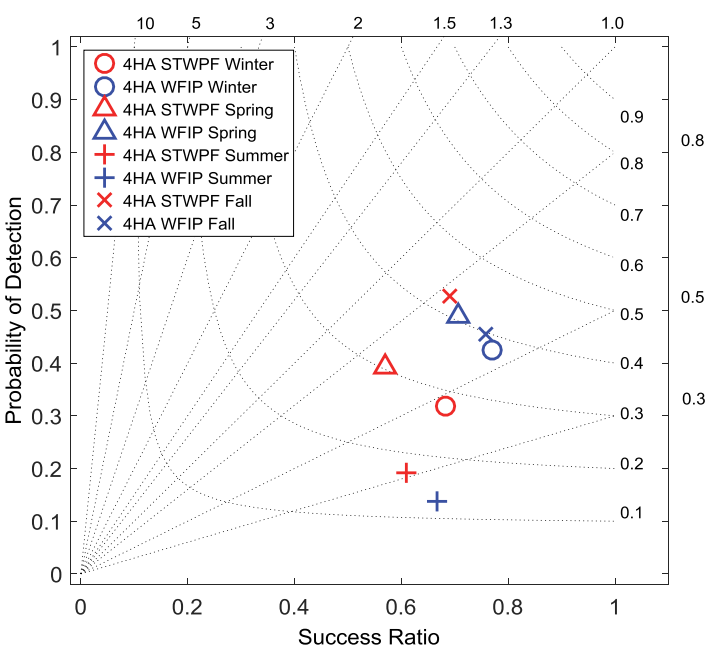

(b) Ramp Definition 2: Ramp magnitude and duration

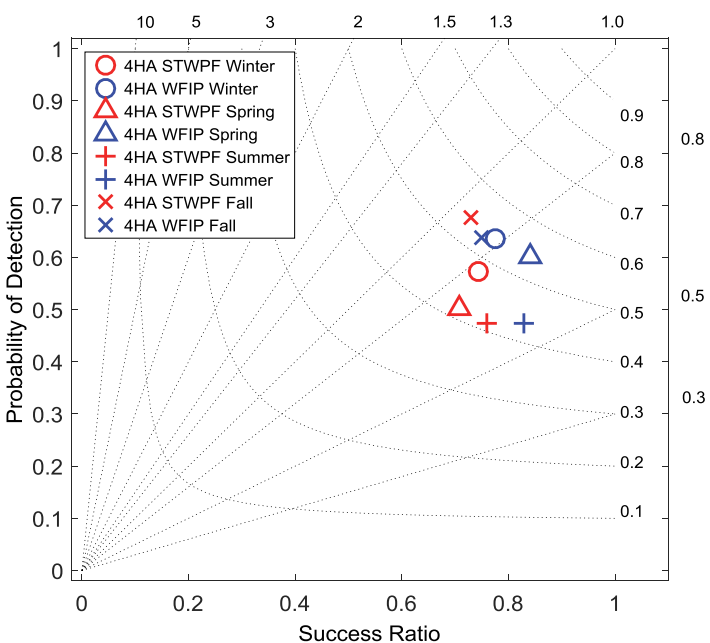

(c) Ramp Definition 3: Ramp rate (d) Ramp Definition 4: Ramp direction, magnitude, and duration 


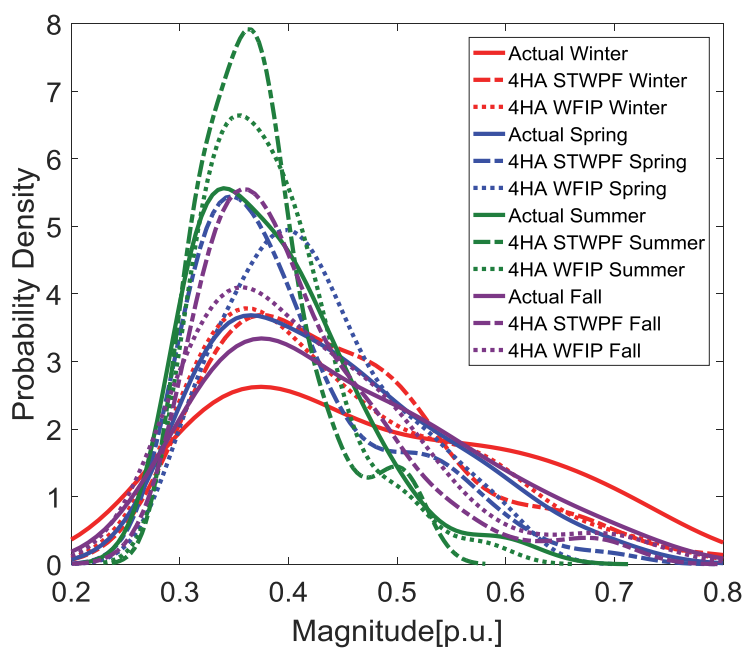

(a) Ramp Definition 1: Ramp magnitude only

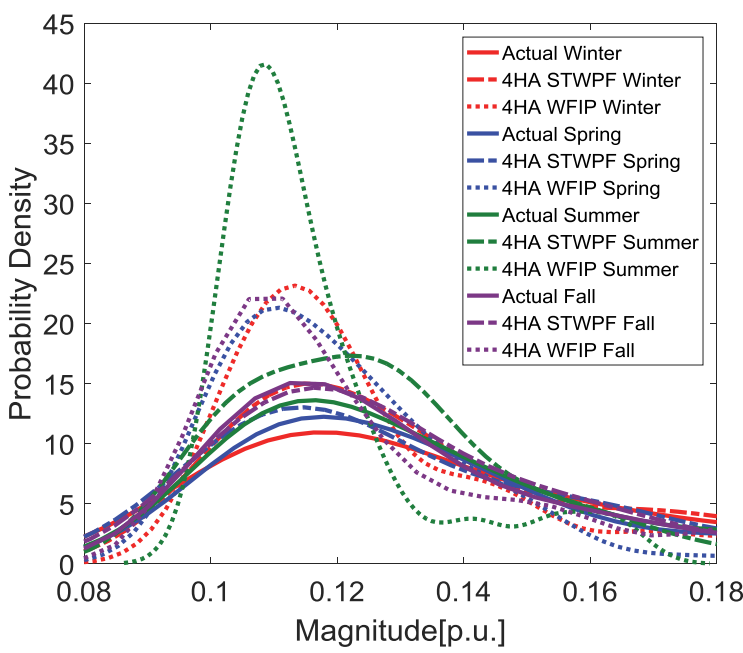

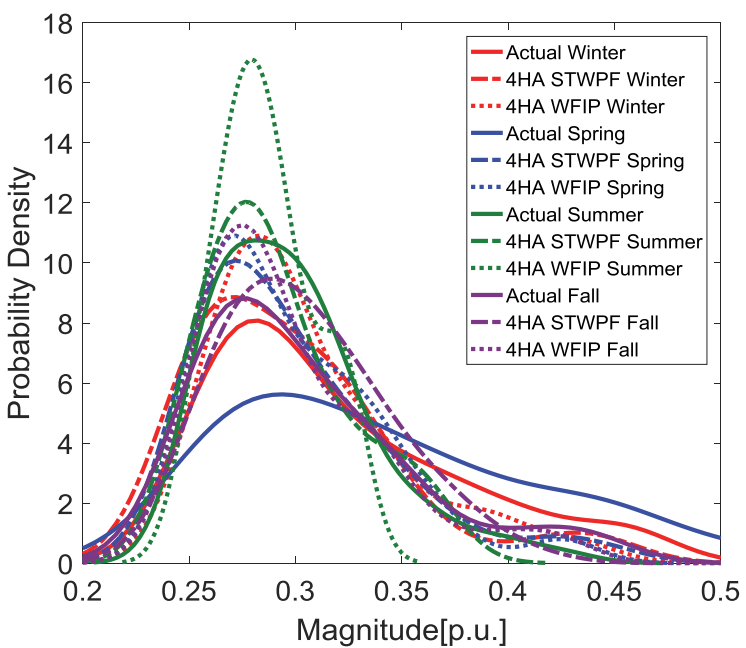

(b) Ramp Definition 2: Ramp magnitude and duration

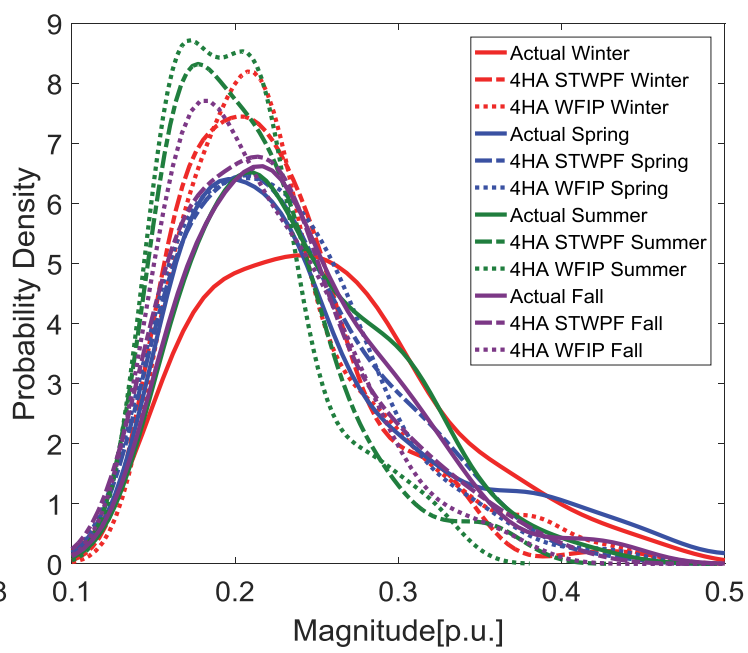

(c) Ramp Definition 3: Ramp rate $\quad$ (d) Ramp Definition 4: Ramp direction, magnitude, and duration Figure 11. Comparing distribution of ramp magnitude between STWPF and WFIP for 4HA forecasts 


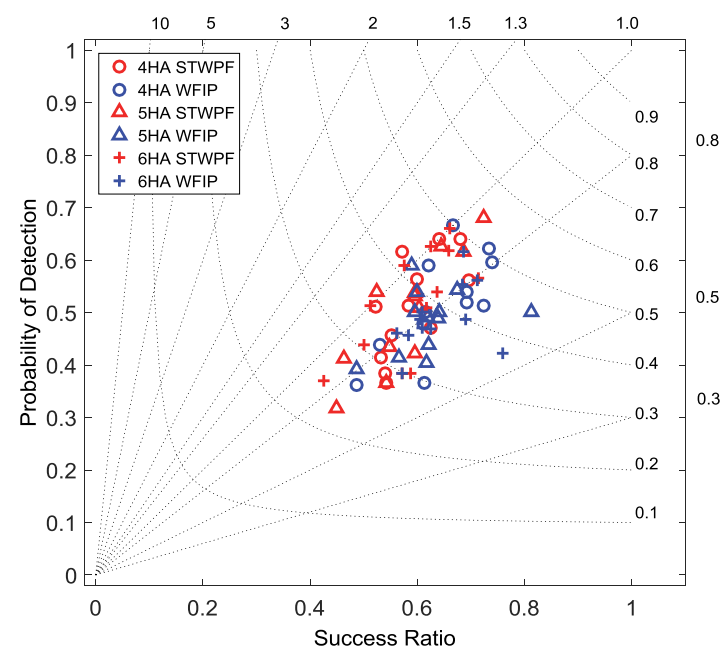

541

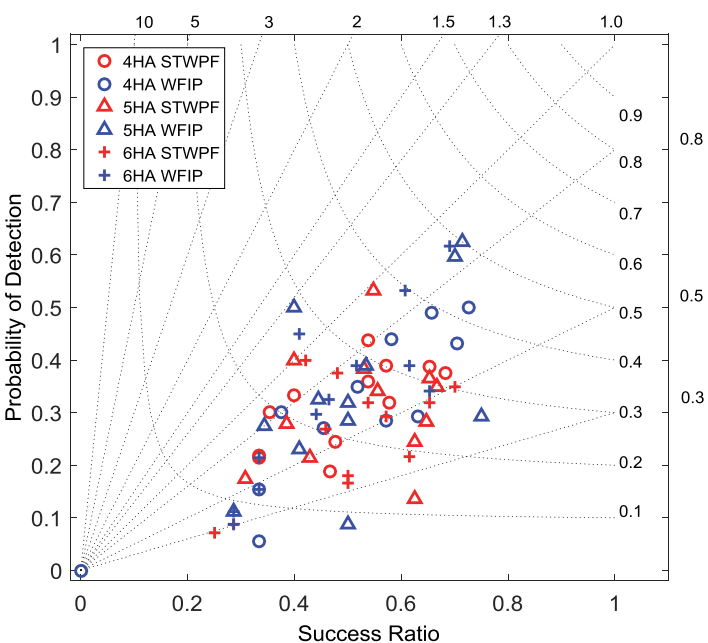

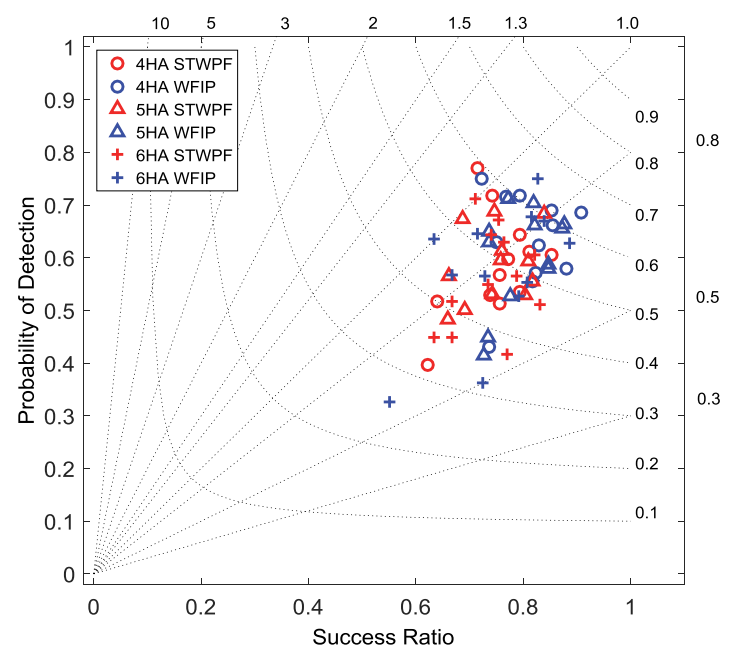

(b) Ramp Definition 2: Ramp magnitude and duration

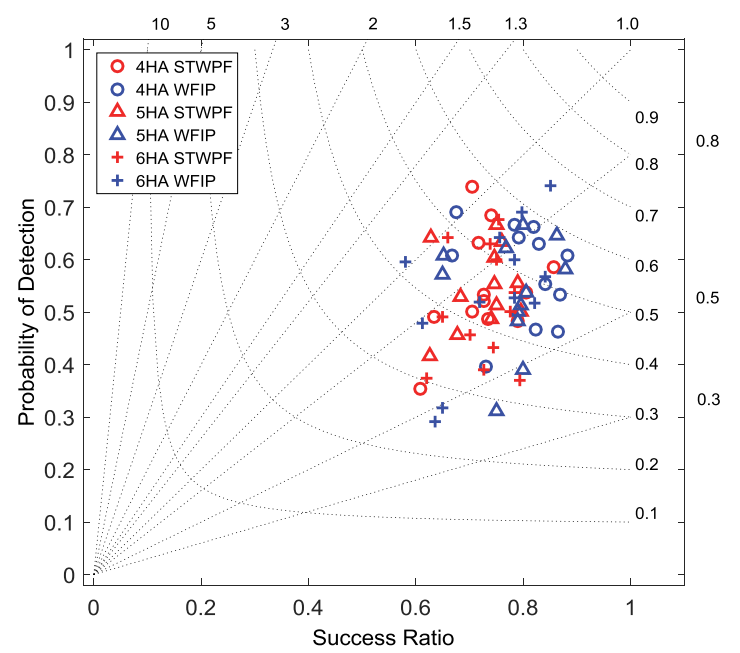

(c) Ramp Definition 3: Ramp rate $\quad$ (d) Ramp Definition 4: Ramp direction, magnitude, and duration Figure 12. Comparison of monthly ramp forecasting performance of STWPF to WFIP for different forecast timescales and ramp definitions 


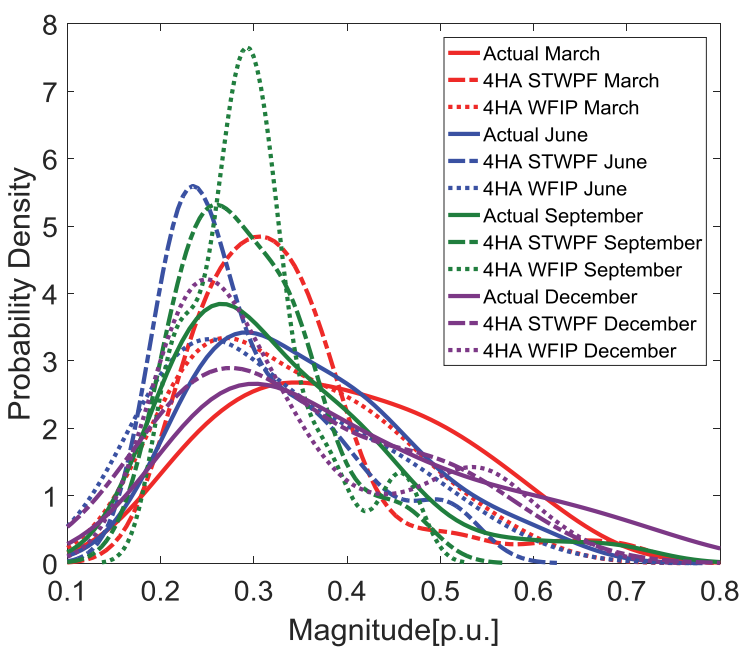

(a) Ramp Definition 1: Ramp magnitude only

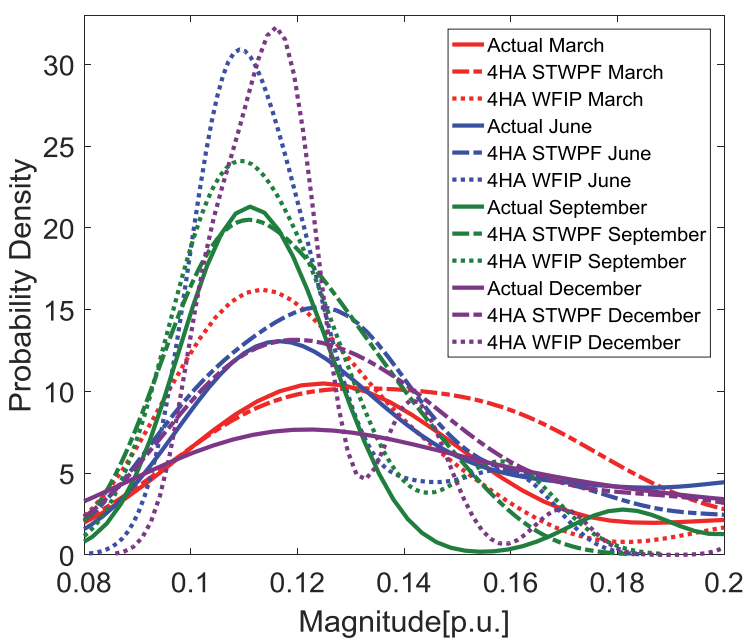

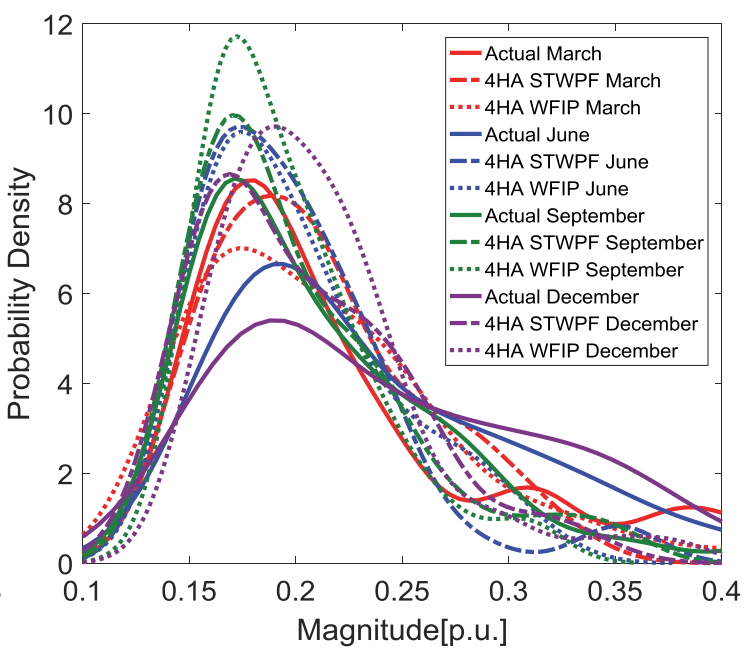

(b) Ramp Definition 2: Ramp magnitude and duration

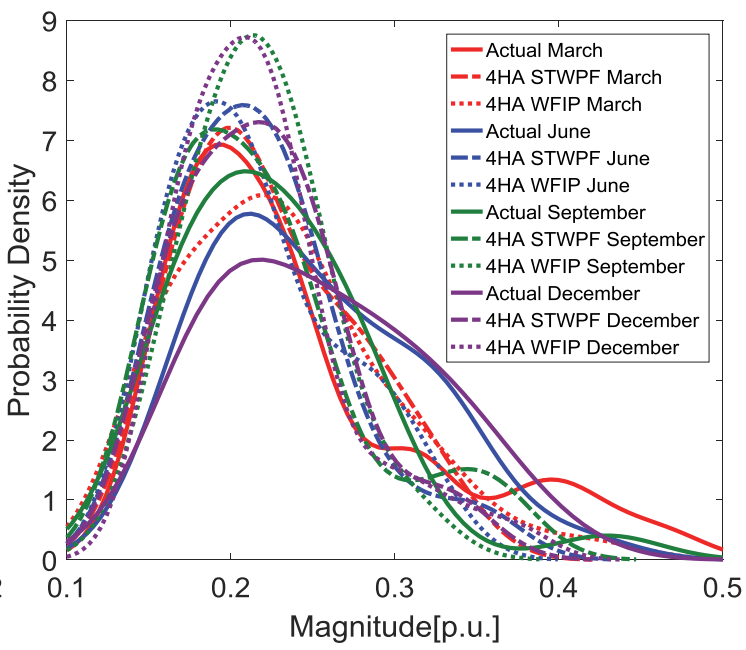

(c) Ramp Definition 3: Ramp rate

(d) Ramp Definition 4: Ramp direction, magnitude, and duration 